\title{
Aspects géomorphologiques des crues des 3 et 4 octobre 1960 dans la partie occidentale du Massif Central
}

\author{
Geomorphological aspects of \\ the 3rd and 4th October 1960 floods \\ in the western Central Massif
}

\author{
PAR \\ R. HLAVEK \\ ET \\ C. LECARPENTIER \\ INGÉNIEUR DU GÉNIE RURAI \\ AU SERVICE TECHNIQUE CENTRAL DE L'HYDRAULIQUE \\ DIRECTION GÉNÉRALE DU GÉNIE RURAL ET DE L'HYDRAULIQUE AGRICOLE \\ MINISTÈRE DE L'AGRICULTURE
}

\begin{abstract}
Conséquences, différentes suivant les bassins ou les groupes de bassins, de précipitations exceptionnelles, mais homogènes.

Distinction de secteurs en fonction de l'activité géomorphologique et suggestions d'aménagements ou d'améliorations permettant de réduire les conséquences des crues :

$1^{\circ}$ L'activité intense et généralisée dans les secteurs de la Corrèze, en entier, et de la Creuse, de sa source d̀ la Rochette, suggère des aménagements d'ensemble;

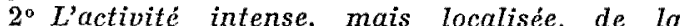
Creuse à l'aval de la Rochette, de la Tardes, de la Voueize et de quelques affluents de la HauteDordogne suggère des aménagements de détail. $3^{\circ}$ Les secteurs de submersion où il $y$ eut une «crue liquide» pourront être protégés par les travanx d'amélioration des secteurs amont.
\end{abstract}

The effects of exceptional homogeneous precipitation on various basins or groups of basins. Identification of sectors in terms of geomorphological activity; suggested developments and improvements to reduce flood effects:

1) The intense general activity along the whole course of the Corrèze and in the Creuse from its source to La Rochette indicate the need for overall remedies.

2) The intense local activity of the Creuse below La Rochette, of the Tardes, the Voueize, and of several tributaries of the Upper Dordogne seem to require local measures;

3) The areas subject to flooding, in which a "liquid flood" occurred, could be protected by improvements to the upstream sectors.

tre de Géographie appliquée de l'Université de Strasbourg nous assura le concours de Mlle A.R. Hirsch, collaborateur technique, et de M. J.

Khobzi, étudiant de $3^{\mathrm{e}}$ cycle.
Les observations ci-après résultent d'une mission effectuée du 14 au 26 octobre 1960 dans les régions touchées par les crues catastrophiques des 3 et 4 octobre, mission pour laquelle le Cen-

\section{I. - CARACTÊRES GÉNÉRAUXX}

\section{DES CRUES DES 3 ET 4 OCTOBRE 1960}

Ces crues furent caractérisées d'une part par leur grande extension, et d'autre part par la disproportion existant entre les hauteurs d'eau observées, qui furent partout très fortes, et l'activité géomorphologique qui ne fut importante que dans certains secteurs seulement.
L'extension du phénomène résulta des conditions météorologiques étudiées en détail par M. P. Fontaine et M. P. Guillot.

La faiblesse relative de l'activité géomorphologique fut tout d'abord due aux conditions naturelles : relief peu accentué et végétation 
généralisée, ce dernier facteur ayant surtout pour effet de réduire le débit solide; à ces causes naturelles s'ajoute enfin l'action de l'homme, essentiellement sous la forme des barrages réservoirs qui atténuèrent généralement la crue et parfois même l'absorbèrent intégralement.

Bien que ces caractères généraux recouvrent de multiples nuances et que la crue n'ait pas eu partout les mêmes effets, il existe une sorte d'affinité géomorphologique entre certains bassins versants que nous examinerons successivement en suivant le rebord occidental du Massif Central, du nord au sud, et en groupant lesdits bassins par affinité géomorphologicque.

\section{II. - ETUDE DE DIVERS BASSINS VERSANTS}

Les divers cours d'eau qui prennent naissance dans le Massif Central, ainsi que leurs affluents, peuvent être groupés comme suit :

II.1. Bassin du Cher : la crue du Cher ne fut pas exceptionnelle et fut essentiellement due aux apports de la Tardes et de son affluent la Voueize. Ces deux cours d'eau, d'altitude moyenne assez faible, n'ont charrié que peu de matériel solide et les dégâts ne furent importants que là où les ouvrages enjambant le lit présentaient des débouchés nettement insuffisants; le mécanisme de destruction des ponts fut généralement le suivant: surverse de la chaussée, puis attaque des culées et des murs de protection, mis à nu par la destruction préliminaire de la chaussée.

II.2. Bassin de la Creuse : nous avons pu étudier cette rivière depuis sa source jusqu'à son confluent avec la Gartempe, soit un cours de quelque 180 kilomètres. La diversité des terrains traversés (Ia Creuse parcourt 120 kilomètres en terrains cristallins avant d'entrer dans les terrains sédimentaires) ainsi que la différence d'importance et de nature de ses aflluents, nous ont amené à distinguer les secteurs suivants :

II.2.1. LE HAUT BASSIN, DEPUIS LA SOURCE JUSQU'A FELLETrN : Ce secteur fait partie du plateau de Millevaches, et l'on n'y trouve guère de bassins de remblaiement. Dès les premiers kilomètres, la rivière s'enfonce, le cours est rapide, mais les dégâts furent peu importants et limités à des engravements aux quelques endroits où la vallée s'élargit en petits bassins. Quelques décollements de versants ont pu être observés, là où la végétation faisait défaut.

II.2.2. De Felletin a Saint-Martial : à partir de Felletin, les dégâts deviennent plus importants; les versants, plus raides, ont favorisé un ruissellement diffus intense, entraînant de nombreux décollements de terrains et permettant aux petits affluents de charrier du matériel solide; mais les dégâts les plus importants se produisirent à Aubusson, en conséquence de l'activité des divers cours d'eau suivants :

- le ruisseau du Fot et son affluent, canalisés dès l'entrée dans Aubusson, engravèrent rapi- dement les égouts qui furent rompus, d'où destruction de la chaussée et propagation brutale du flot liquide;

- la Creuse a gravement endommagé ses quais, ainsi que les chaussées et immeubles de la partie basse de la ville;

- enfin la Beauze, pénétrant dans la ville par deux sinuosités, a encore accentué ces dernières et les deux grands sapements qui se sont produits ne laissent pas d'être inquiétants quant à leur évolution future; la confluence de la Beauze et de la Creuse est particulièrement défavorable et nous $\mathrm{y}$ voyons l'explication de la destruction du pont d'accès à l'usine des lampes Fala (croquis $\mathrm{n}^{\circ} 1$ ).

Remarquons dès maintenant que seule la Beauze eut une activité géomorphologique, les dégâts dus à la Creuse et au Fot résultant surtout de l'endiguement ou de la canalisation des eaux.

En aval d'Aubusson, les dégâts se généralisent, la vallée étant encaissée entre des versants raides, de $30^{\circ}$ à $45^{\circ}$. Une certaine activité géomorphologique se manifesta sur les versants (décollements, cônes de déjection à la sortie de thalwegs non fonctionnels). D'une manière générale, les sinuosités ont été recoupées, avec dépôts

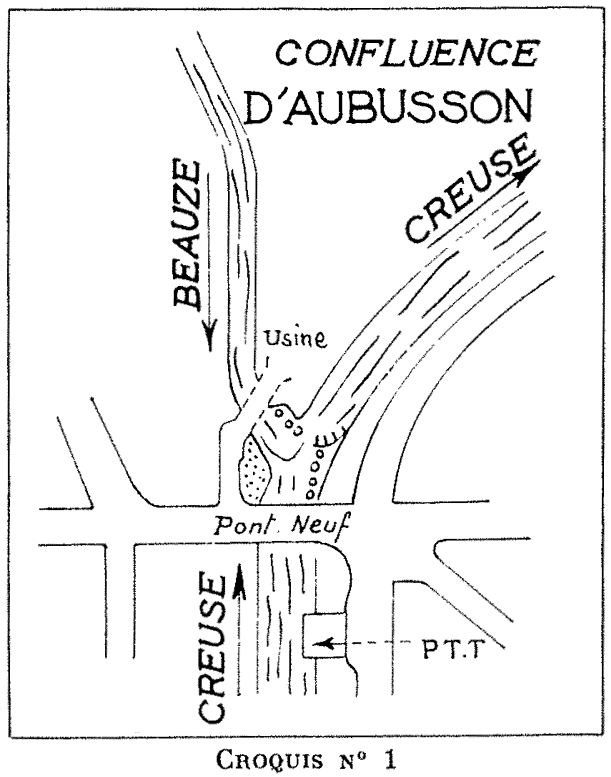




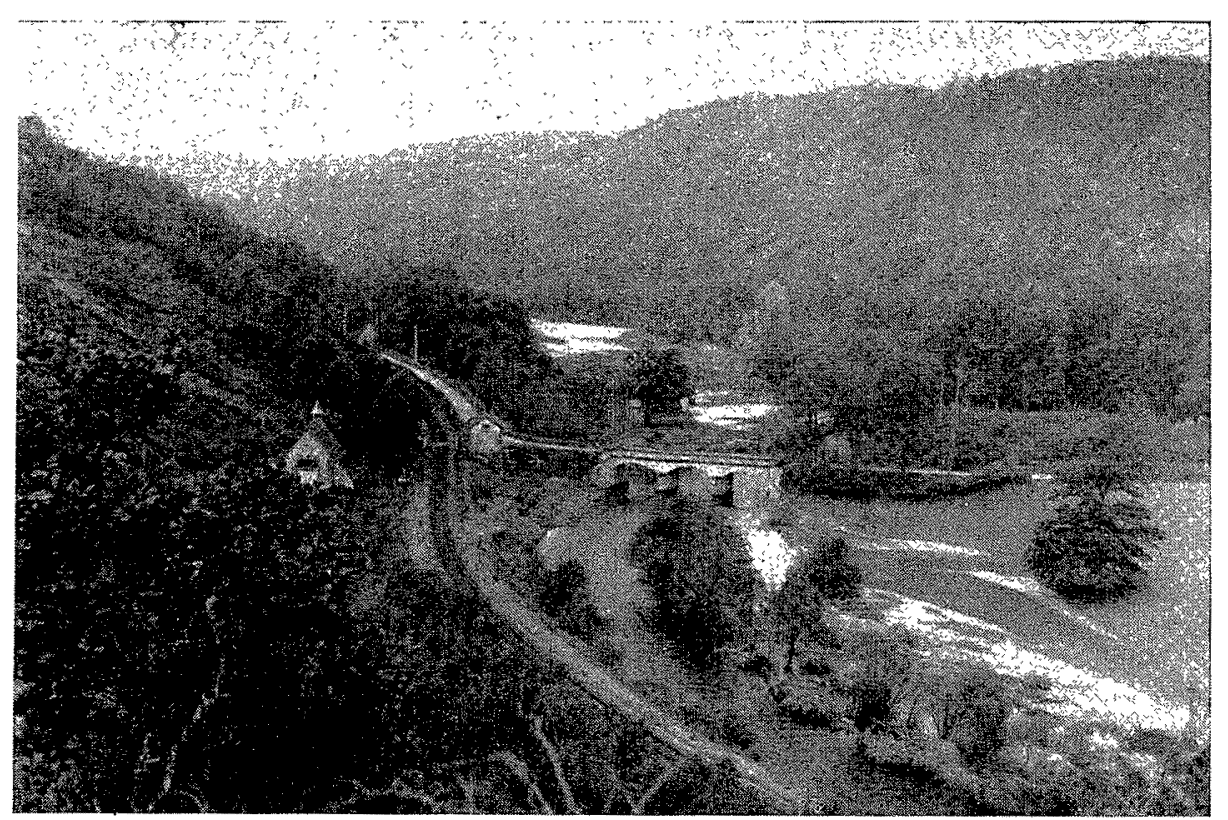

Рното 1

La Creuse entre Aubusson et Alleyrat (vue vers l'amont depuis la N.142) : au centre de la photo, le pont de la D.18 dont la photo 13 donne une vue détaillée.

d'atterrissements (photo 1 ) ou même creusement de chenaux de crue (photo 2).

II.2.3. De Saint-Martial a Glenic : ce secteur est caractérisé par l'absence d'affluents notables et la diminution du ruissellement intermédiaire. La Creuse se présente comme une succession de sections rétrécies, avec des sapements, et de bassins où la crue s'est étalée, laissant parfois quelques engravements (photo 3 ).

II.2.4. Secteur DES Gorges, dE Glenic AU MENoux : dans ce secteur l'érosion des versants fut très faible et le courant rapide, l'eau n'ayant fait que passer, parfois cependant avec violence (la Celle-Dunoise).

La Petite Creuse subit elle-même une crue importante, mais les dégâts se limitèrent aux abords des ponts, et les engravements furent peu importants.

Depuis le confluent de la Grande et de la Petite Creuse, et jusqu'en amont d'Argenton, les effets de la crue ont été entièrement conditionnés par les barrages (Eguzon, la Roche-bat-l'Aigue, la Roche-au-Moine) et à un moindre degré par les installations riveraines (canaux d'amenée des moulins en particulier); en général les sapements et les engravements furent localisés à l'aval des rapides et des barrages (photos 4 et 5 ).

II.2.5. La vallée Moyenne, du Menoux a LA Roche-Posay : à l'aval du Menoux, la Creuse quitte le massif cristallin pour entrer dans les
Pho'To 2

Ebauche de chenal de crue et engravements (la Creuse à la Rochette) : la rive gauche, concave, présente des versants assez raides au pied desquels coule la Creuse, alors que la rive droite, convexe, constitue une terrasse alluviale qui se raccorde progressivement aux versants et qui a été fortement engravée, notamment dans laxe du chenal de crue.

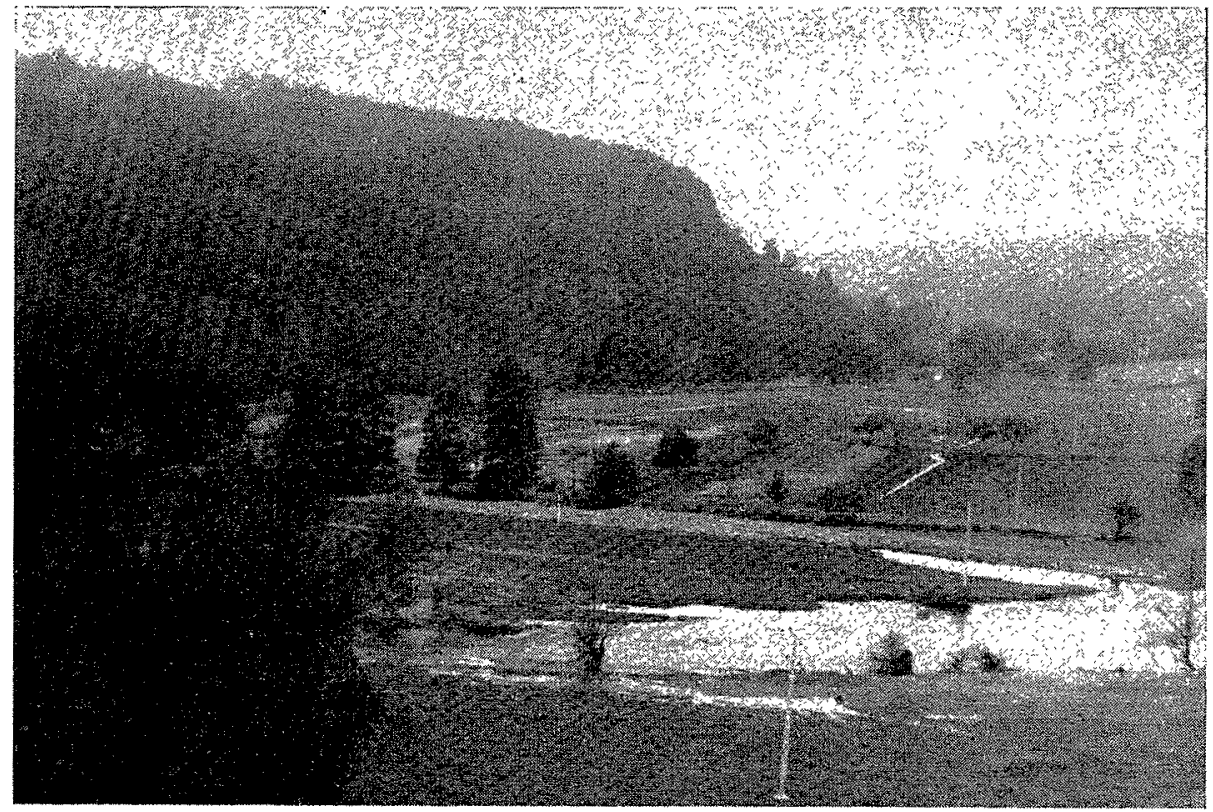


Рното 3

Ebauche de chenal de crue et épandage de matériel (pont de la D.4 sur la Creuse entre SaintLaurent et Pionnat).

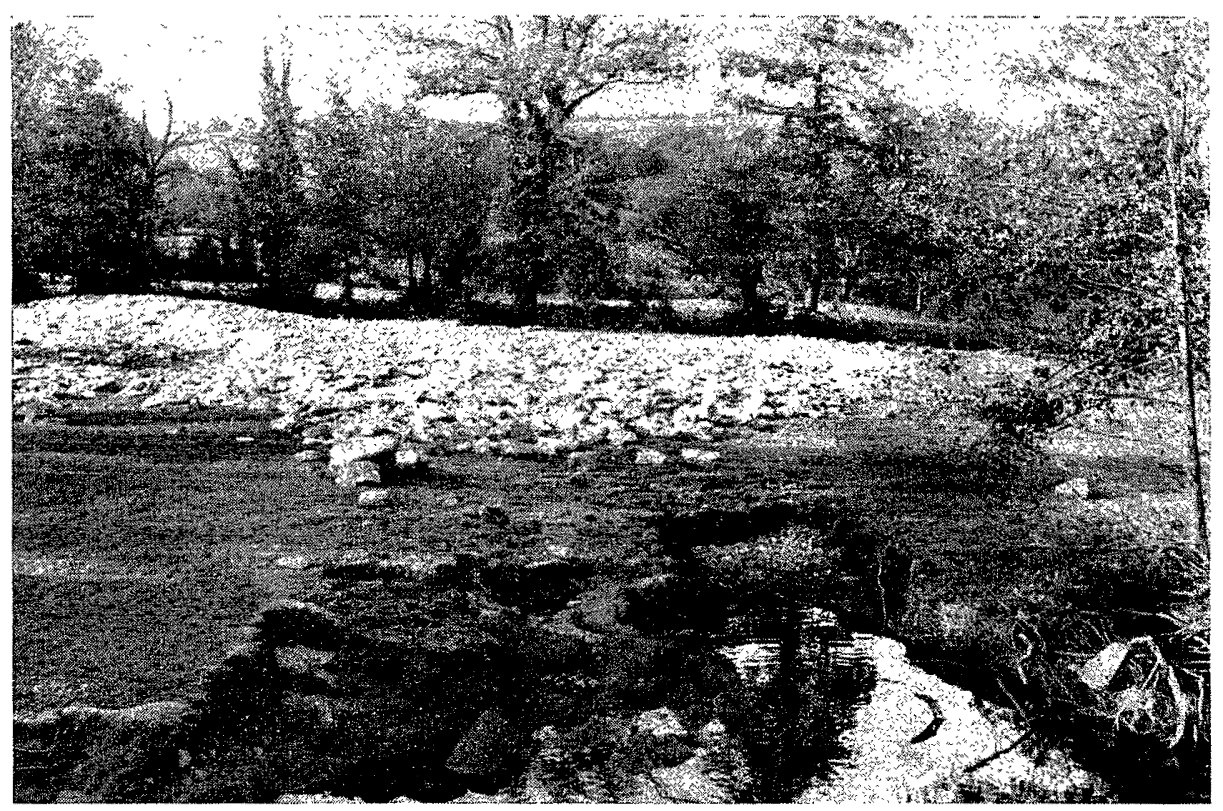

A l'aval de La Roche-Posay, marquant le confluent de la Gartempe et de la Creuse, la crue n'a pratiquement plus laissé de traces.

II.3. Bassin de la Vienne : les dégâts ayant été pratiquement négligeables à l'aval de SaintJunien et même dès Limoges, nous n'avons étudié que le haut bassin de la Vienne et ses affluents, soit de l'amont vers l'aval la Combade, la Maulde et le Thaurion.

Bien que ces cours d'eau aient été soumis à des précipitations très importantes, les crues correspondantes ne furent pas, toutes proportions gardées, aussi catastrophiques que celles de la Corrèze et même de la Vézère.

Une première explication peut facilement être

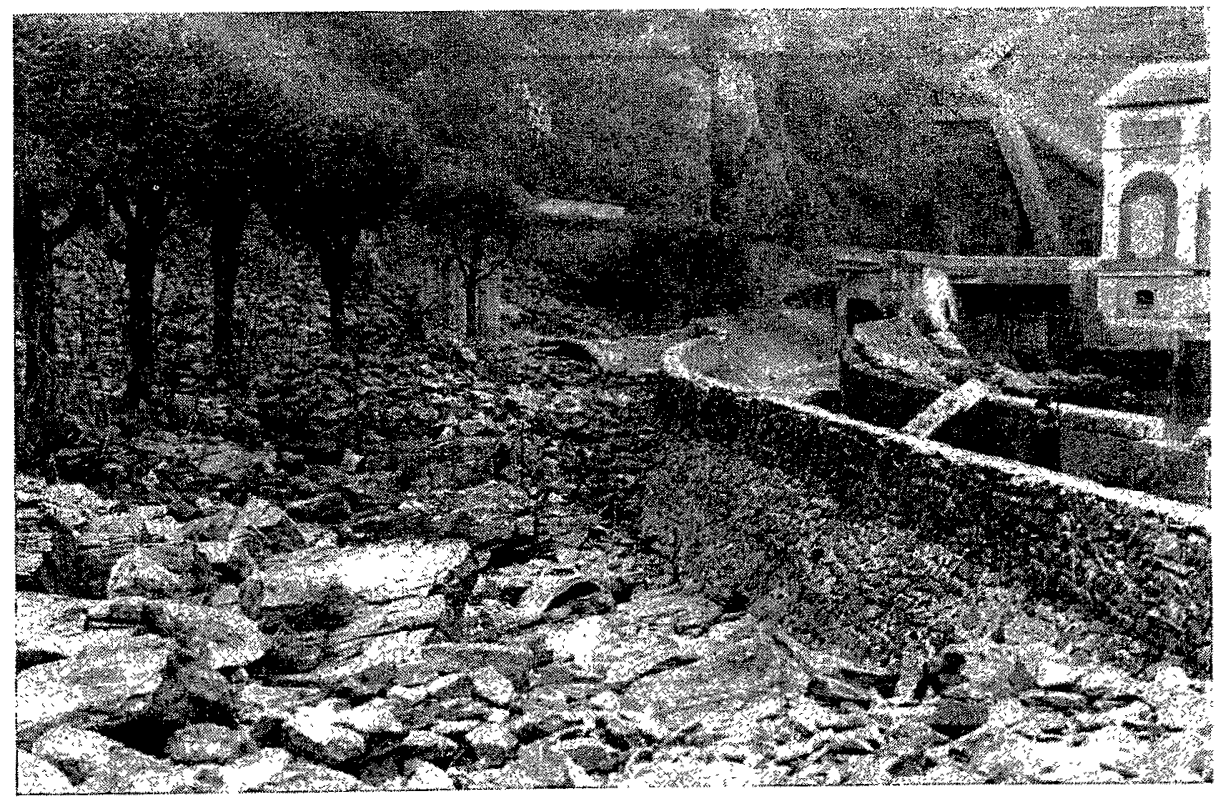

Pното 4

Barrage d'Eguzon, sur la Creuse: vue du canal de fuite. 
Рното 5

La Creuse à l'aval du barrage de la Roche-au-Moine cà l'amont du pont de la D.38 allant de Baraize au Pin); on voit à l'arrière-plan le sapement de la terrasse caillouteuse menaçant la route qui conduit au barrage; au centre de la photo, on remarque la situation du limnigraphe après la crue; l'amorce du nouveau chenal est visible au premier plau.
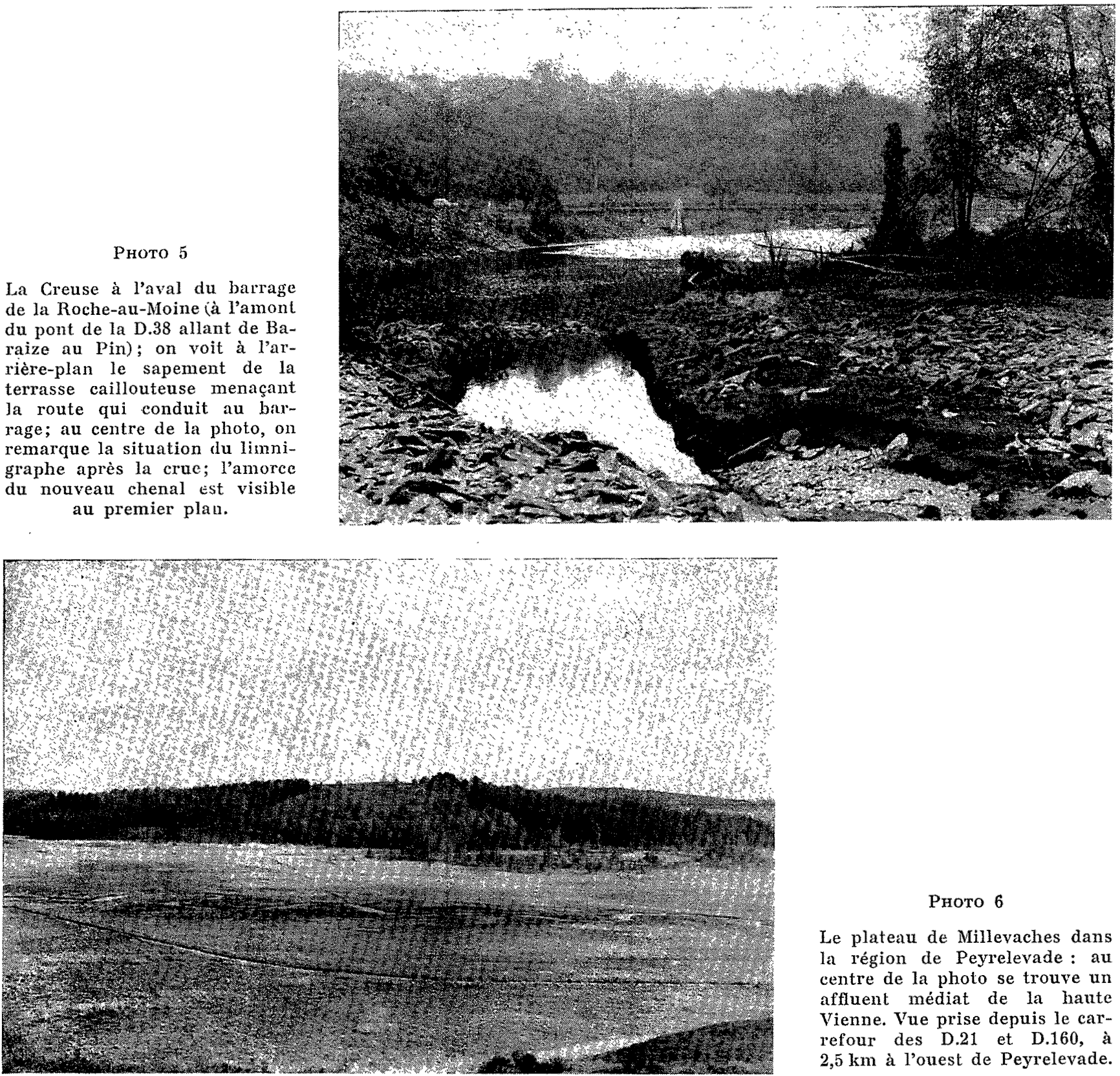

Рното 6

Le plateau de Millevaches dans la région de Peyrelevade : au centre de la photo se trouve un affluent médiat de la haute Vienne. Vue prise depuis le carrefour des D.21 et D.160, à $2,5 \mathrm{~km}$ à l'ouest de Peyrelevade.

trouvée dans le rôle régulateur joué par les nombreux barrages qui coupent les hautes vallées de ces cours d'eau, et plus particulièrement le Thaurion et la Maulde. Une deuxième explication tient à ce que l'ensemble de ces cours d'eau est issu du plateau de Millevaches, dont le relief assez mou (photo 6) et les sols spongieux à forte capacité de rétention sont de nature à ralentir le ruissellement et à limiter le débit solide, générateur des gros dégâts.

II.4. Bassin de la Dordogne : notre mis sion fut limitée à l'examen des affluents de rive droite du haut bassin de cette rivière, et nous distinguons successivement :
II.4.1. LA VÉZÈre A L'AMONT DU CONFLUENT AVEC LA CoRrÈze : à l'amont d'Uzerche, la Vézère n'a guère causé de dégâts qu'à Treignac (photo 7), cette haute vallée bénéficiant des circonstances favorables indiquées pour la Vienne au paragraphe ci-dessus. A l'aval d'Uzerche, nous avons tout d'abord le secteur des «Gorges de la Vézère» où l'activité géomorphologique paraît avoir été très faible sinon nulle; ce n'est qu'après son entrée dans le bassin de Brive, au Saut-du-Saillant, que les dégâts deviennent plus importants et sont dûs essentiellement à une submersion plus ou moins prolongée, sans phénomène d'érosion ou de dépôt.

II.4.2. LA CoRrÈzE : c'est dans le bassin de la 


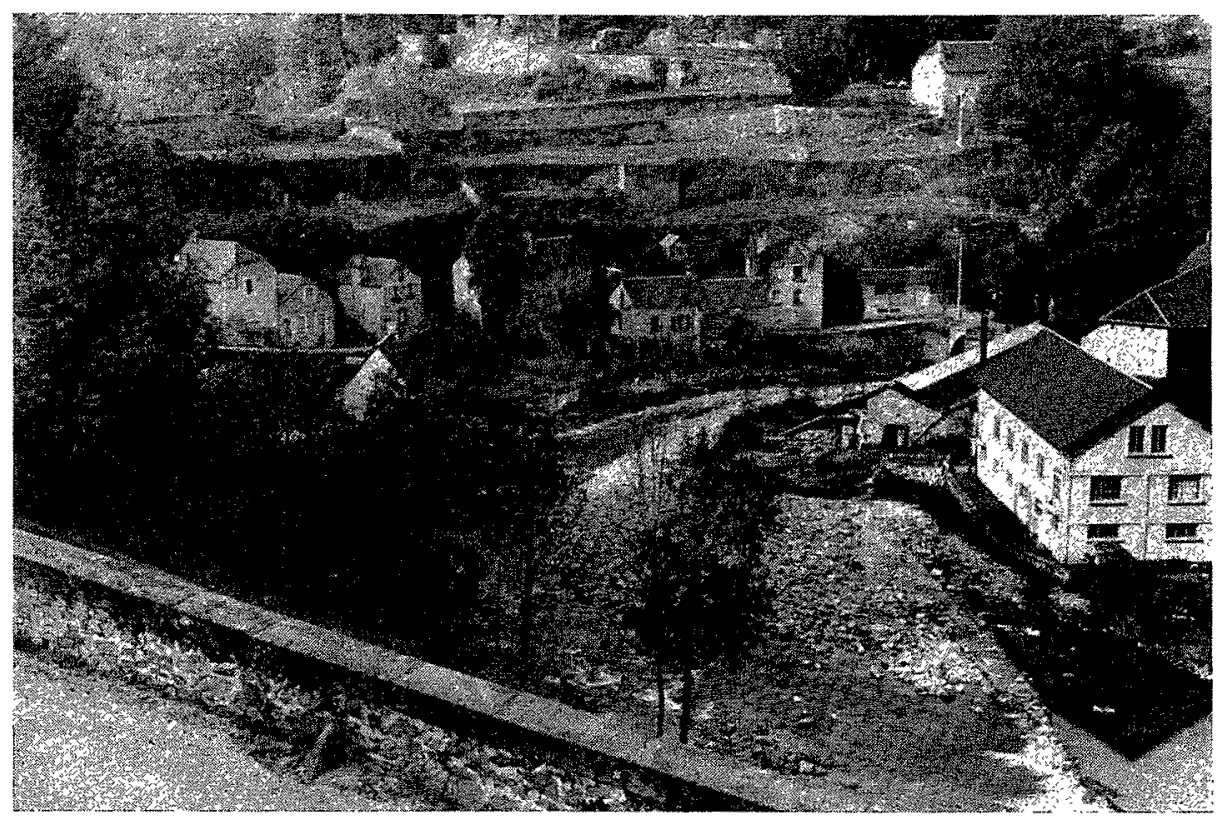

\section{Рното 7}

La Vézère à Treignac : au premier plan à droite, on distingue le seuil oblique prolongé par les ouvrages de prise et de décharge.

\section{Рното 8}

La Corrèze à l'amont du confluent avec la Vimbelle : on ne distingue que l'extrémité aval des chenaux, qui sont bien anastomosés, mais de faible profondeur et se poursuivent par un épandage de galets. (La rivière coule, à l'arrière-plan, de gauche à droite.)

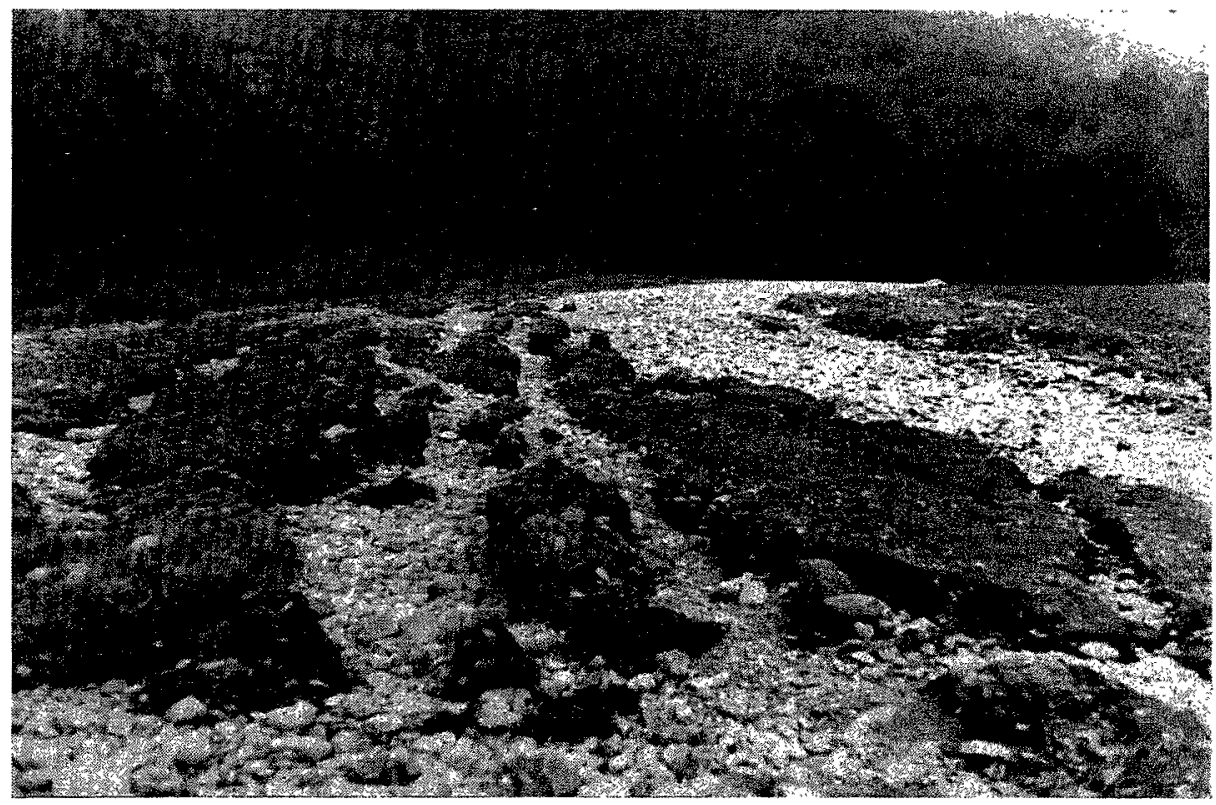

Corrèze, et plus particulièrement le long de celleci, que se sont produits les dégâts de loin les plus importants, qu'il s'agisse de dommages urbains (Tulle et Brive) ou de résultats d'une activité géomorphologique intense et généralisée sur les versants et dans les fonds de vallée (Vimbelle et Corrèze).

On peut invoquer lout d'abord une cause météorologique : les précipitations exceptionnelles ne se sont en effet pas seulement abattues sur le plateau de Millevaches, mais aussi sur les monts des Monédières et en contre-bas sur la région de Tulle et le massif qui la domine au sud, la Roche-de-Vic.

D'autres causes peuvent être recherchées dans les caractéristiques des bassins versants, à sa- voir d'une part que le haut bassin de la Corrèze ne s'enfonce que très peu dans le plateau de Millevaches, et d'autre part que le réseau hydrographique converge vers Tulle, la Corrèze recevant en effet, à quelques kilomètres à l'amont de Tulle, son principal affluent, la Vimbelle, puis aux abords de Tulle des affluents tels que la Montane, l'Avalouze, et la Ganette.

Enfin, nous n'oublierons pas de remarquer que, mis à part le modeste barrage de Bar (entre le village de Corrèze et Tulle), aucune retenue n'existe sur le bassin versant de la Corrèze, qui garde une forte pente naturelle.

Les dégâts se résument ainsi de l'amont vers l'aval :

a) en amont du bassin de Tulle : l'activité 


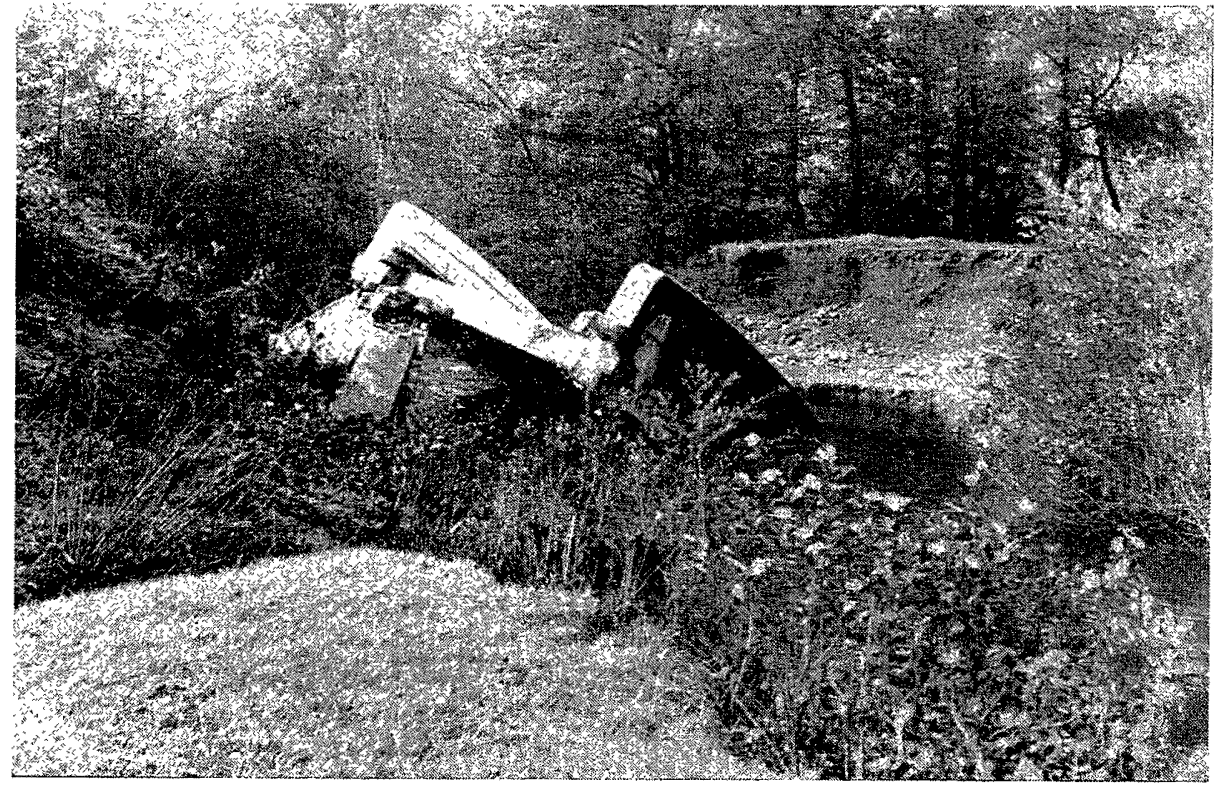

Рното 9

L'Avalouze (ou Saint-Bonette) au pont de la D.29, près de Saint-Bonnet-sur-Avalouze. Ors remarquera que la culée de rive droite a complètement disparu. géomorphologique fut intense tant sur les versants, particulièrement dans les Monts des Monédières, que dans les fonds de vallée où se sont produits des sapements et des engravements dans les sections élargies du cours de la Vimbelle et de la Corrèze; cette dernière a d'une manière générale recoupé ses sinuosités et les dégâts ont pu être spectaculaires et importants (photo 8), mais sont restés surtout agricoles.

b) du bassin de Tulle au bassin de Brive: les dégâts s'accentuent encore dans ce secteur; les phénomènes de versants furent nombreux (arrachements, coulées boueuses, foirages de talus): les affluents de la Corrèze furent très actifs, qu'il se soit agi de l'Avalouze (photo 9), de la Ganette ou de la Montane.
A Tulle même, les dégâts furent d'une importance considérable, la ville étant établie au débouché d'un rétrécissement prononcé, et le cours de la rivière étant endigué en un chenal se resserrant progressivement pour former un véritable entonnoir; de nombreux embâcles se sont formés, entraînant parfois des ruptures d'ouvrages transversaux au lit.

A la sortie de Tulle, la Corrèze a très violemment sapé ses berges particulièrement au «Monument des $99 \gg$ (photo 10) et au Pont de Mulatet (photo 11 et croquis $n^{\circ} 2$ ). Plus en aval, dans le rétrécissement qui fait suite au bassin de Tulle, le passage de la crue a provoqué le « ramonage» du fond de la vallée, souvent jusqu'à la roche en place; dans ce secteur les dégâts sont nom-

\section{Phoro 10}

Le «Monument des $99 »$, à Tulle: le sapement, en rive droite concave, menace encore le monument ainsi que quelques maisons et jardins en amont.

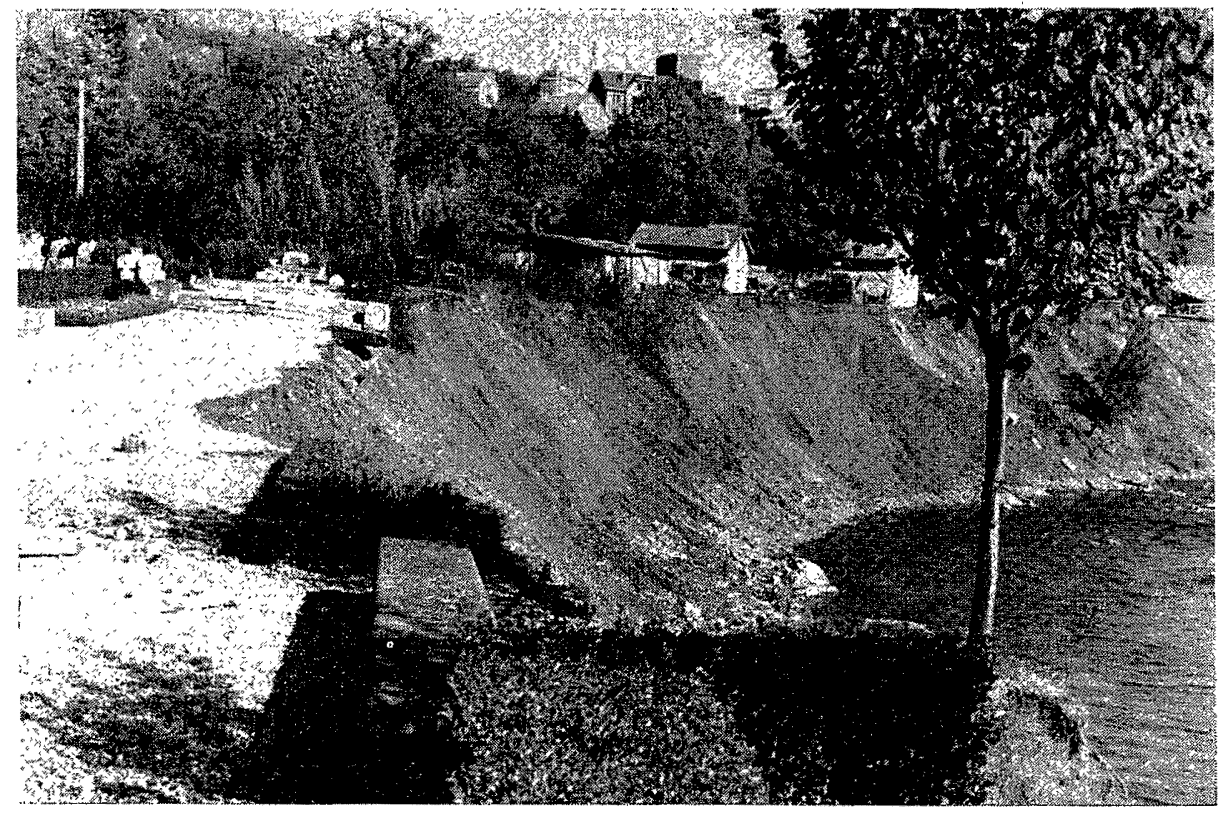




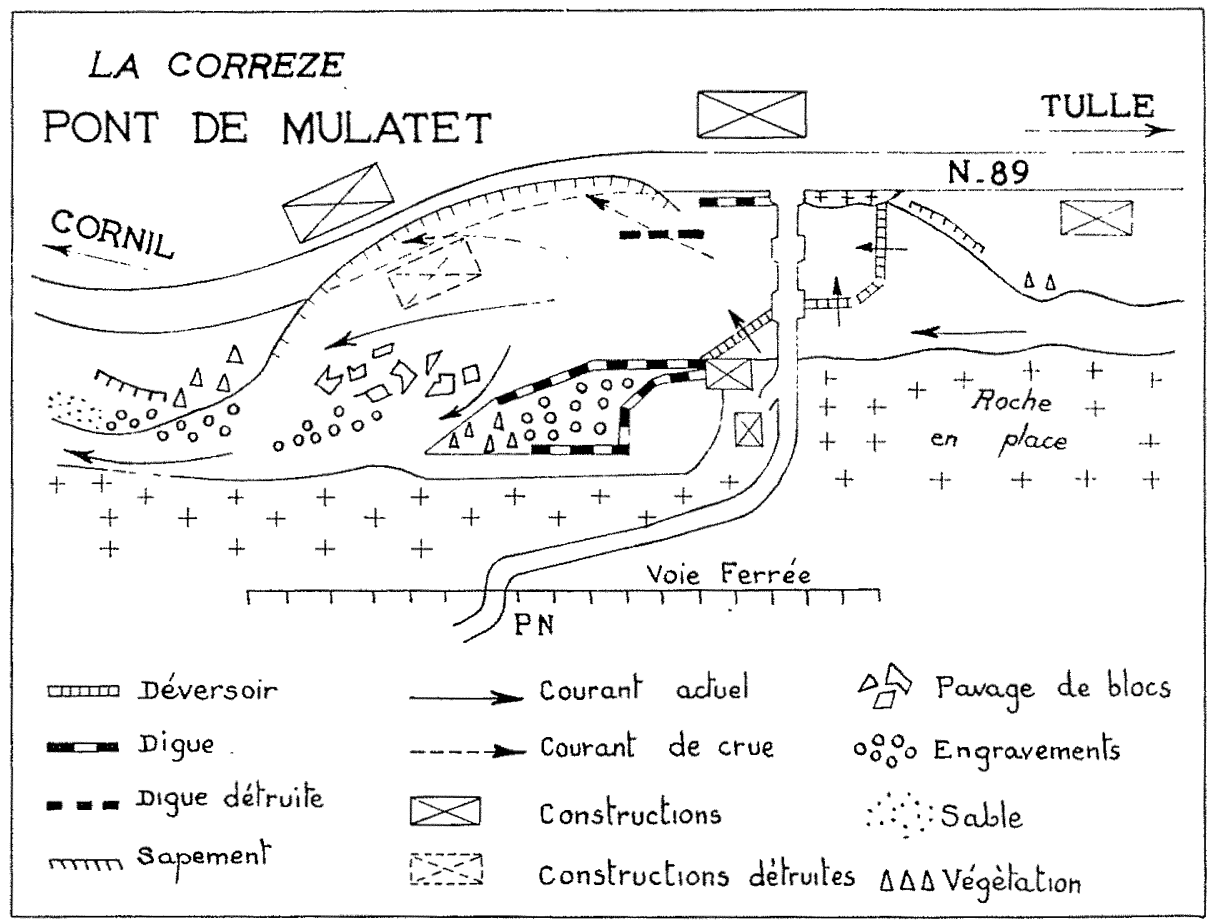

breux : maisons emportées, ponts effondrés, digues contournées, route et voie ferrée sapées.

c) le bassin de Brive : dès l'entrée dans ce bassin, la crue a changé de caractère, devenant essentiellement «liquide 》 et les dégâts résultèrent généralement d'une submersion importante et prolongée, parfois aggravée par la rupture des protections habituelles (exemple du hameau des Granges et de la rupture du remblai de la voie ferrée de Saint-Yrieix).
II.4.3. LA VÉzère A L'AVAL DU CONFLUENT AVEC LA CorrìzF : ici encore la crue a été « liquide » (cf. : §II.4.1 in fine et $\S$ II.4.2-c) et s'est étalée largement dans le fond de vallée, quelques sapements s'étant produits dans les secteurs où la vallée se rétrécit.

II.4.4. LES AFFluents DE LA DORdogne A LAMONT DE BEAULıEU : les dégâts ont généralement été négligeables sur ces affluents, tous de rive droite, essentiellement en raison de l'exis-

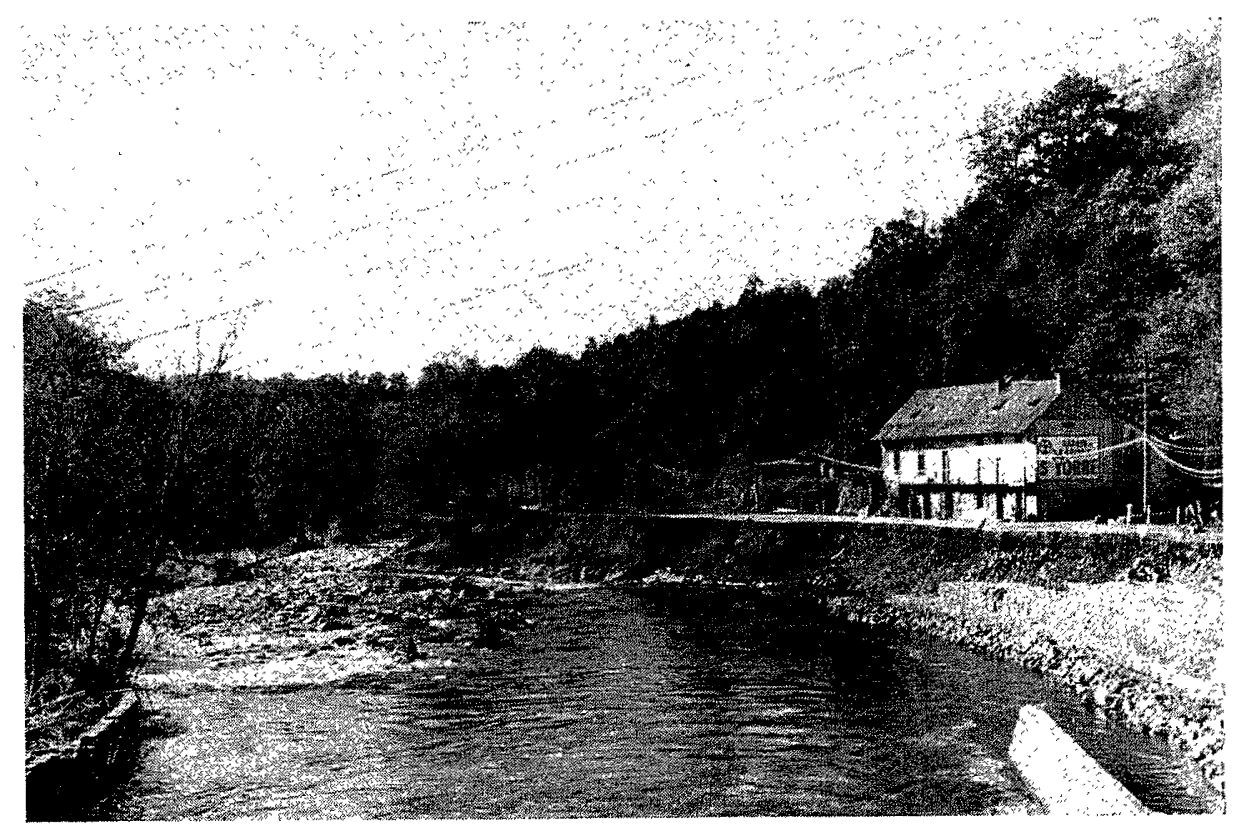

Рното 11

Recul de berge par sapement (la Corrèze au pont de Mulatet). Cf. croquis $n^{\circ} 2$. 


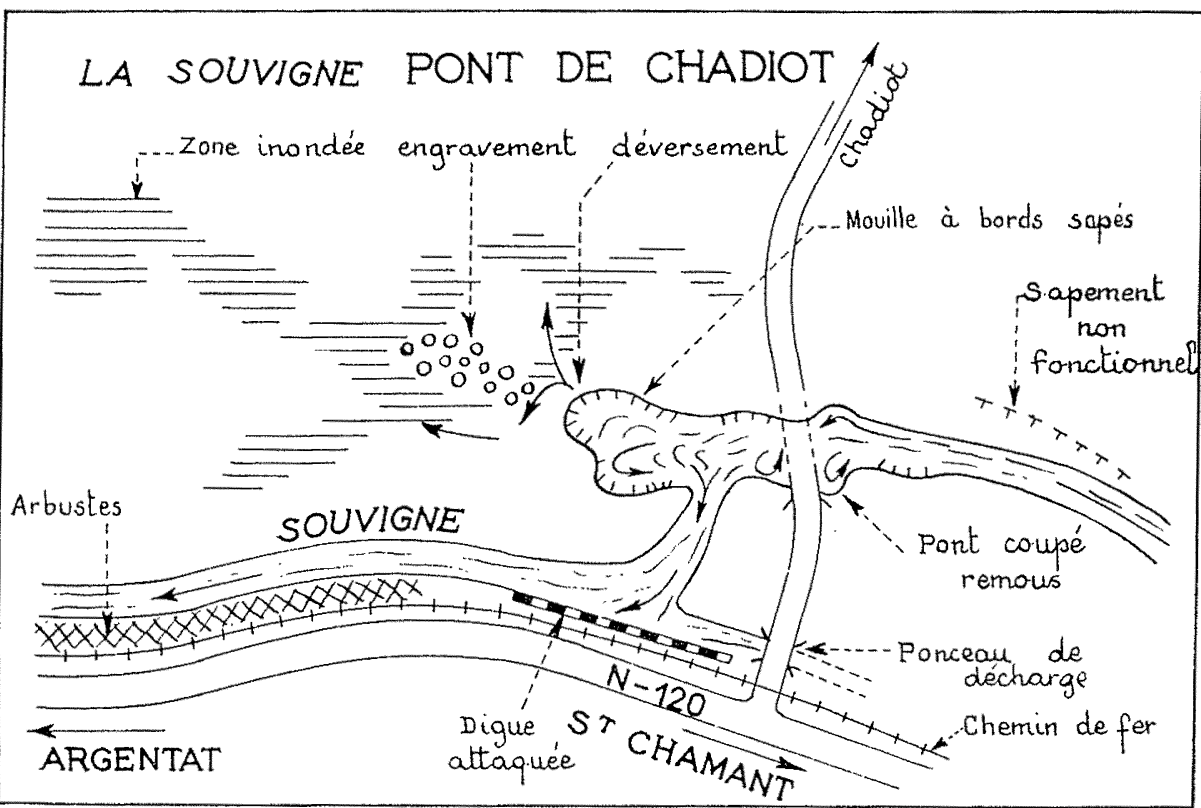

tence de nombreux barrages-réservoirs; tel est le cas des cours d'eau suivants : Liège, Diège, Triouzoune, Luzège, et Doustre; pour la Liège et la Diège, il importe de signaler qu'elles sont de plus issues du plateau de Millevaches.

Par contre, des dégâts plus importants furent signalés et observés sur deux cours d'eau non aménagés : la Souvigne et la Ménoire. Pour la Souvigne, il semble qu'un ròle fondamental doive être attribué à son affluent de rive droite, la Franche Valène, issue du massif de la Rochede-Vic, dont les versants raides furent soumis à d'intenses précipitations; cette rivière présente de plus un cours constitué par une succes- sion de méandres et de sinuosités parfois désastreuses (cf. : croquis $\mathrm{n}^{\circ} 3$ ). La crue de la Ménoire fut de même importance, mais par suite de l'encaissement de la vallée, les dégâts furent plus localisés et généralement limités aux destructions d'ouvrages (cf. : croquis $\mathrm{n}^{\circ} 4$ ).

II.4.5. LE CÉou : ce cours d'eau est un de ceux qui, quọique affectés par les mêmes précipitations, sont assez éloignés du centre de la zone des crues catastrophiques pour que leur étude doive être entreprise séparément, et rattachée à celle de cours d'eau tels que le Vert et le Celé, affluent du Lot. Le temps nous a manqué pour commencer une telle étude.

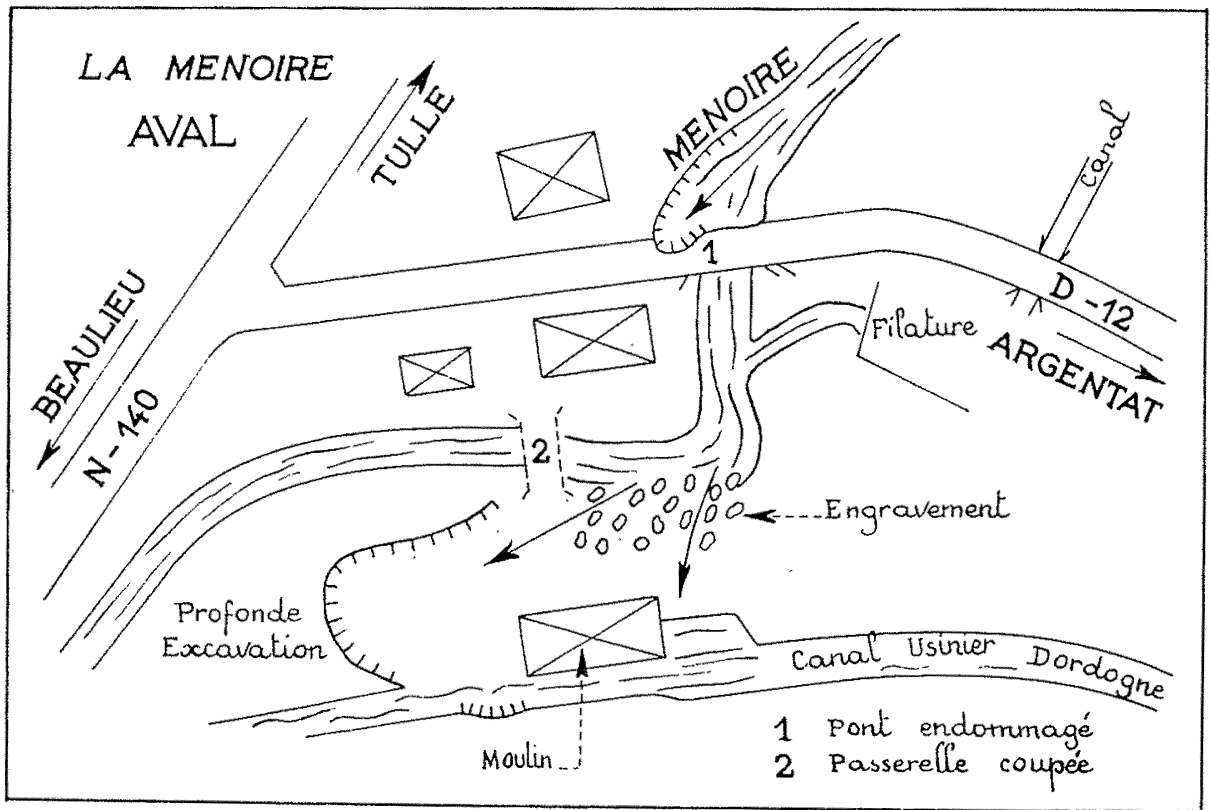




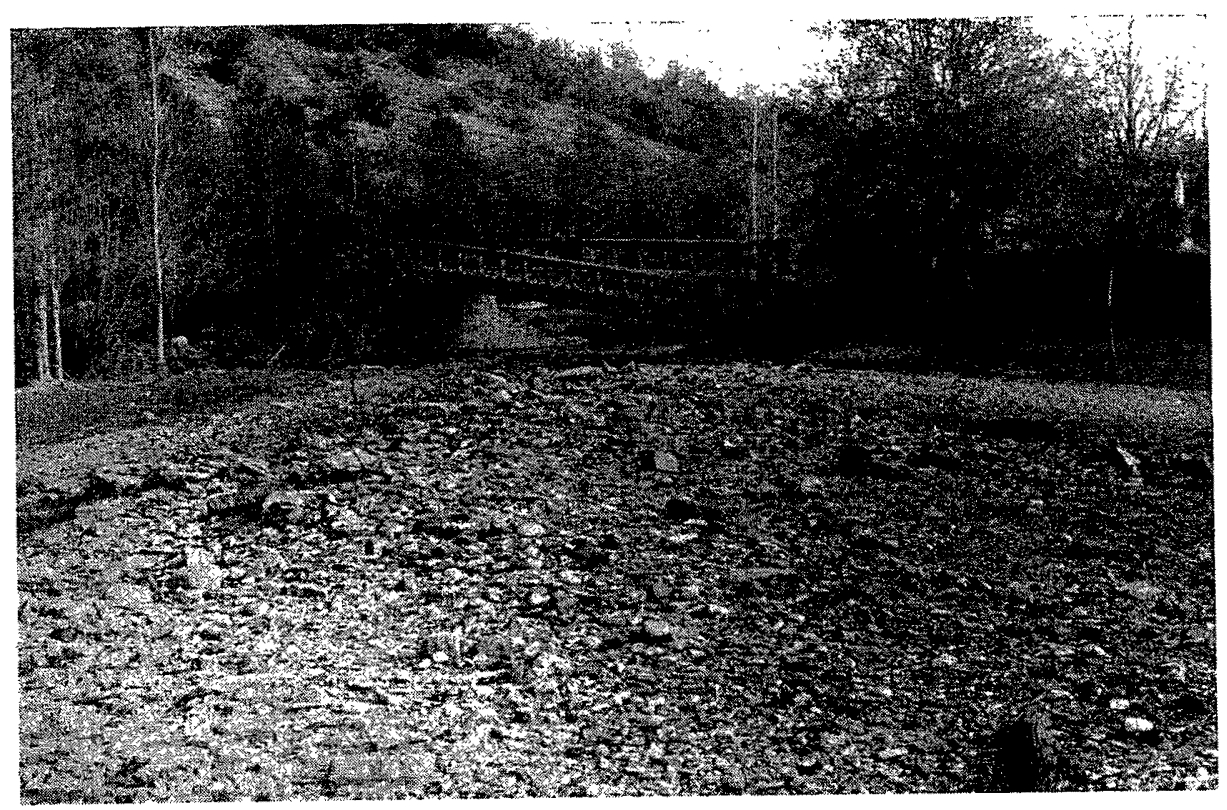

Pното 12

Le Céou, au pont du Vo vers Gaumiers (soit à $2 \mathrm{~km}$ environ au sud de Saint-Martial de $\mathrm{Na}$ birat); il y a eu sapement en rive gauche, à l'amont du pont, avec affouillement de la culée, d'où basculement du tablier, et à l'aval du pont, épardage de matériel visible au premier plan.

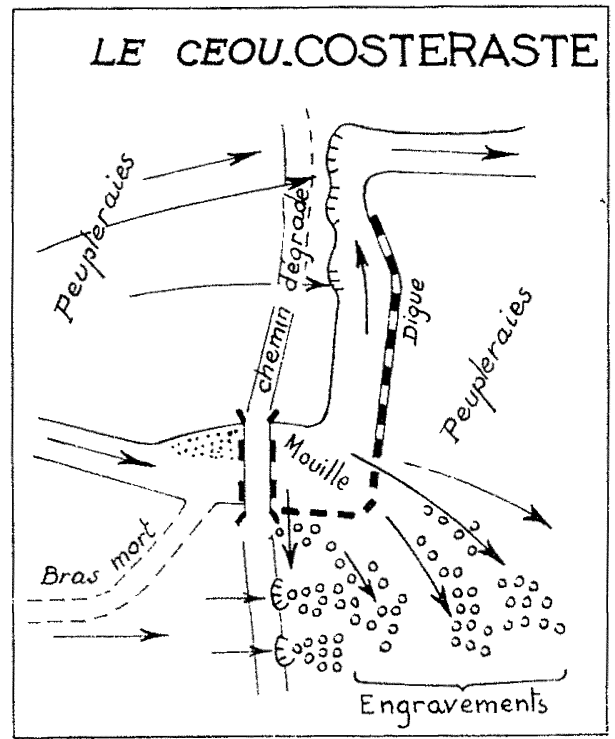

\section{III. - ETUDES DES DIVERS DEGATS}

Au lieu d'ètudier, comme nous venons de le faire, les dégâts dans leur contexte géomorphologique, c'est-à-dire dans le cadre de chaque bassin ou partie de bassin, nous pouvons essayer de les classer d'après leur nature, ce qui nous amène à distinguer d'une part les "dégâts naturels », entrant dans le cadre de l'évolution normale des cours d'eau, et d'autre part les « dégâts anthropiques » résultant de l'installation par l'homme d'ouvrages enjambant ou bordant les cours d'eau; la place nous manquant pour développer cette question (cf.: mémoire original déposé à la S.H.F.), nous laisserons de côté les «dégâts naturels» qui ressortent des descriptions ci-dessus pour passer rapidement en revue Croquis $N^{\circ} 5$ les «dégâts anthropiques》.

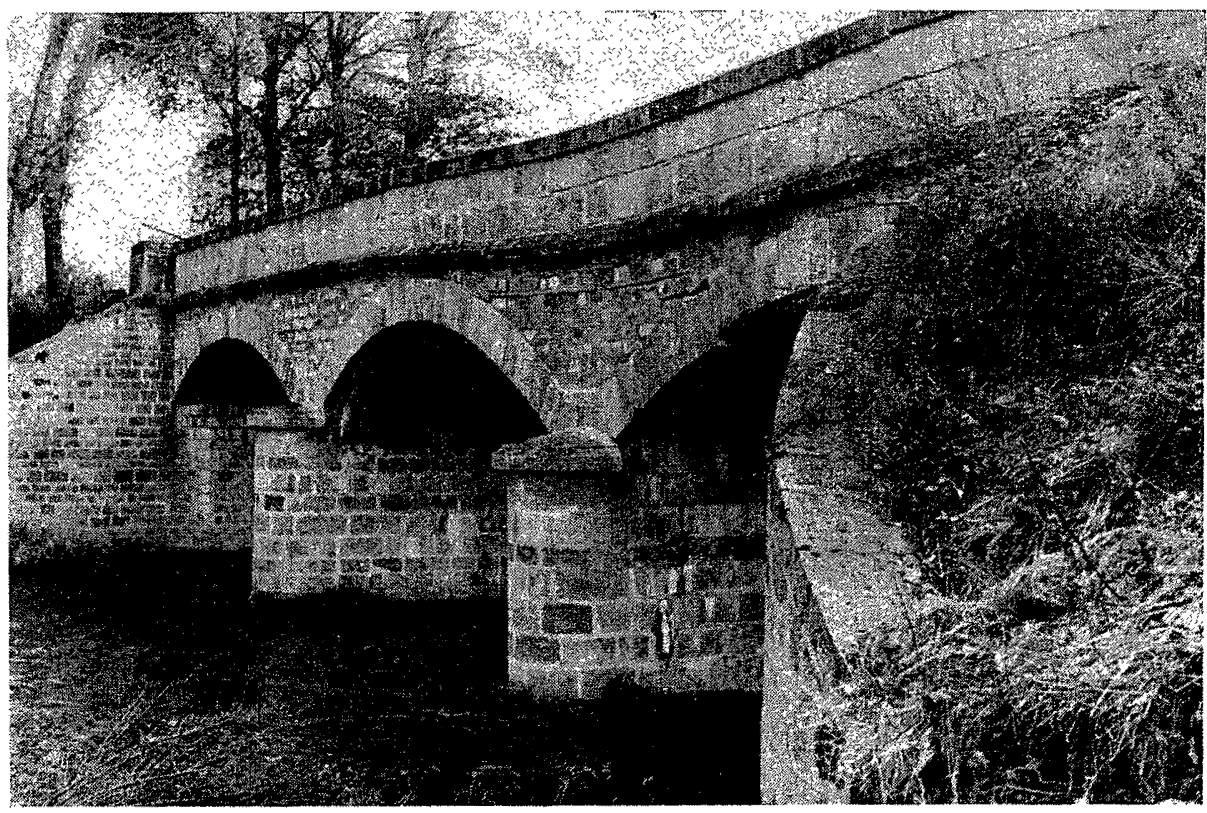

Phoто 13

Affouillement de pile (pont de la D.18 sur la Creuse, entre Aubusson et Alleyrat); Ja photo 1 donne une vue d'ensemble de l'aval, alors qu'ici, la photo est prise de l'amont. 
A. - Les ponts : étant entendu que les débouchés étaient insuffisants pour une crue aussi exceptionnelle, il reste à remarquer que si les affouillements de piles ont été rares (photo 13), les attaques des culées et des murs de protection furent très fréquentes, particulièrement pour les ponts établis dans une courbe (photos 9 et 12 , croquis $n^{\circ} 4$ ); des ponts établis perpendiculairement au lit mineur ont pu cependant être endommagés, soit par des remous (croquis $n^{\circ} 3$ ), soit parce que, situés en aval d'une sinuosité, ils se trouvaient en biais par rapport au courant de crue (pont de la N.717 sur la Verneigette, affluent de la Voueize); le croquis $n^{\circ} 5$ représente un exemple de dynamique de remous, semble-t-il particulier au Céou.

B. - Les ouvrages de prise en rivière : il s'agit là à peu près essentiellement des ouvrages de prise et d'amenée d'eau d'anciens moulins, les déversoirs ayant créé à leur aval une zone de torrentialité (croquis $n^{\circ} 2$ et photo 11) et les canaux d'amenée ayant assuré un cheminement facile de la crue (croquis $n^{\circ} 4$ ).

C. - Les endiguements : ont joué un rôle néfaste soit par suite de leur largeur insuffisante, soit par suite d'un mauvais tracé, soit par le jeu simultané de ces deux facteurs.

\section{IV. - CONCLUSIONS}

En ce qui nous concerne, nous pouvons retenir que la caractéristique essentielle de la situation météorologique génératrice de ces crues reste son extension considérable. Il en résulte que les divers cours d'eau étudiés furent soumis à des précipitations dans l'ensemble comparables, les différences d'effets de la crue étant alors essentiellement fonction de la nature des bassins versants. Les distinctions géomorphologiques que nous avons pu établir paraissent donc avoir de ce fait un certain caractère de généralité.

Si nous reprenons alors l'ensemble des cours d'eau, il apparaît possible de distinguer trois types de secteurs, à savoir :

1. les secteurs où l'activité géomorphologique a été importante et généralisée : tels sont le bassin de la Corrèze dans sa totalité et celui de la Creuse de Felletin à La Rochette. Ces secteurs sont justiciables d'aménagements d'ensemble rationnels (traitement des versants et création de barrages-réservoirs par exemple).

2. Les secteurs où l'activité géomorphologique, sans pour autant être négligeable, a été limi- tée à des endroits déterminés. Il est possible que dans ce cas des aménagements de détail s'avèrent suffisants (rectifications de cours, protections des berges, installations et ouvrages d'art). Peuvent se ranger dans cette catégorie : la Tardes et la Voueize, la Petite Creuse, la Creuse des environs d'Ahun à ceux d'Argenton, certains affluents de la Dordogne.

3. Enfin. les secteurs de submersion, c'est-àdire ceux où la crue s'est étalée dans des vallées larges ou relativement larges, comme la Creuse en aval d'Argenton et la Vézère depuis son entrée dans le bassin de Brive. Ces secteurs ont souffert de la hauteur d'eau bien plus que de l'activité géomorphologique. La seule solution semble alors d'aménager la partie amont de ces mêmes rivières et de leurs affluents.

Cette distinction est certes schématique et des études de détail nous conduiraient peut-être à la modifier, certainement à faire intervenir des subdivisions. Nous nous permettons seulement de rappeler que notre communication est le résultat d'une simple et rapide mission de reconnaissance. 